\title{
Recalcitrant livedoid vasculopathy; long-term remission achieved with pentoxifylline combined with colchicine
}

\author{
Fernando SGS ${ }^{1}$, Gamage $\mathbf{N}^{2}$ \\ ${ }^{\prime}$ Base Hospital, Padaviya, Sri Lanka, \\ ${ }^{2}$ Base Hospital Wathupitiwala, Sri Lanka. \\ Correspondence: Dr. Geehan Shanaka Fernando \\ e-mail: geehanshan@gmail.com \\ (D) https://orcid.org/0000-0003-0538-8473 \\ Submitted on 17.06.2020 and accepted for publication on 14.10.2020
}

\section{Introduction}

Livedoid vasculopathy (LV) is a thrombotic cutaneous disease characterized by episodic painful leg ulceration. Ulcers are typically seen around malleoli, which are often bilateral and often heal, leaving marked atrophic and disfiguring scars. The condition is common among young and middleaged women. The exact pathogenesis of LV is unclear. Yet, it is thought to be caused by systemic dysregulation of coagulation, resulting in the formation of fibrin thrombi of the superficial dermal plexus. The main goals of the treatment are pain relief, prevention of recurrent ulceration and progressive scarring. Most treatment regimens are based on anecdotal off-label protocols. Despite therapy, long term remission has always been a challenge.

\section{Case presentation}

A 15-year-old female, presented with painful leg ulceration for one year duration. She neither had fever nor other systemic symptoms. Patient had no pertinent medical history including venous thrombosis. Also, she did not have known family history of lupus erythematosus or clotting disorders. Examination revealed tender, multiple discrete superficial to deep ulcers and crusted plaques around malleoli bilaterally. Peri-lesional hyperpigmentation and atrophic stellate scars were also noticed (Figures $1,2,3 \& 4)$. There were no palpable purpura, petechiae, livedo reticularis, livedo racemosa, retiform purpura, subcutaneous nodules, digital necrosis or visible varicose veins. Peripheral pulses were present. Rest of the physical examination and review of systems were negative.

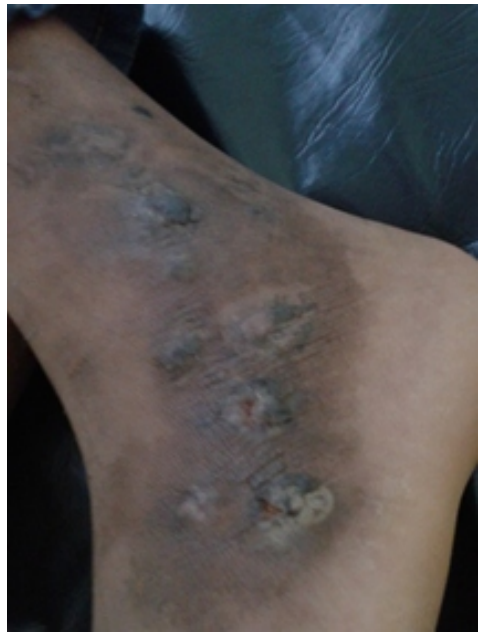

Figure 1: Lesions over lateral side of the ankle (left)

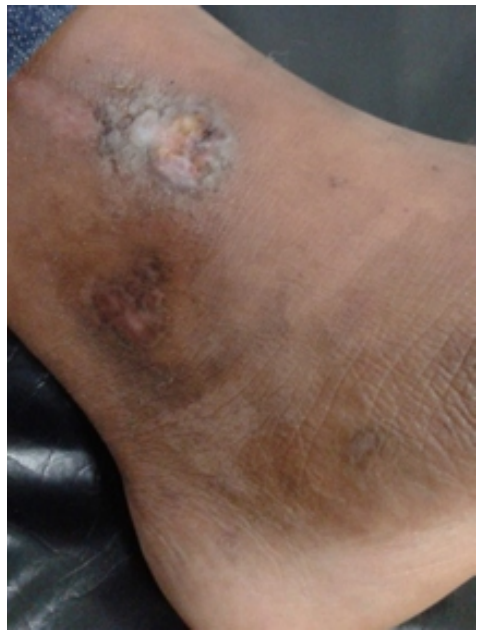

Figure 2: Lesions over medial side of the ankle (left)

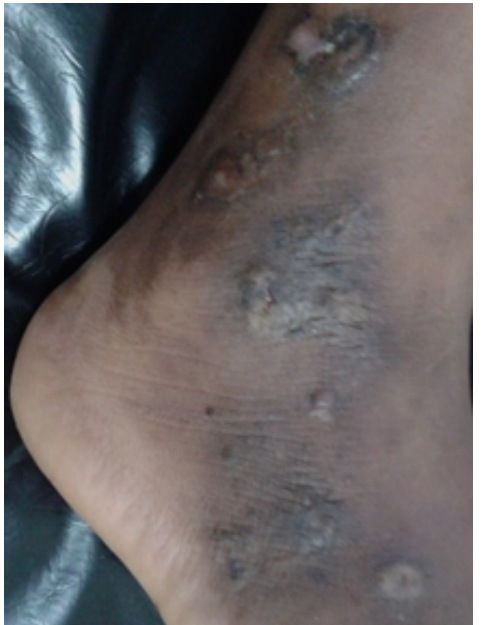

Figure 3: Lesions over the lateral side of the ankle (right) 


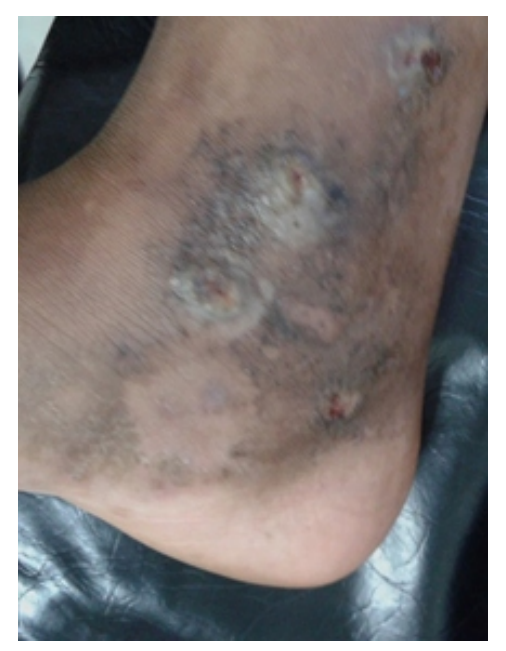

Figure 4: Lesions over medial side of the ankle (right)

Basic investigations including full blood count, erythrocyte sedimentation rate, C-reactive protein, urine analysis, prothrombin time and activated partial thromboplastin time were normal. Moreover, lupus anticoagulant test, anti cardiolipin antibody IgG, antinuclear antibody test and Anti-double stranded DNA antibodies were negative. Factor 5 Leiden, prothrombin 20210G $>$ A and MTHFR $677 \mathrm{C}>\mathrm{T}$ mutations were not detected. Serum homocysteine levels were normal. Serum cryoglobulin were not detected. Venous stasis was excluded by doing a duplex scan of lower limbs.
Skin biopsy taken from medial side of the ankle on left side showed typical histopathological features of livedoid vasculopathy i.e. mild hyperkeratosis and acanthosis of epidermis, thickening and hyalinisation of dermal vessels with evidence of almost obstructed lumen and fibrinoid material contained in some of the vessel walls.

She experienced significant improvement on a regimen of aspirin $75 \mathrm{mg}$ daily and pentoxifylline $400 \mathrm{mg}$ three times daily for one month. Despite this regimen she continued to have mild recurrences at least once in three months. Thereafter, colchicine $0.5 \mathrm{mg}$ twice daily dose was started as an adjuvant therapy, while aspirin was omitted. All her ulcers resolved within three months of aforementioned regimen. Thereafter, pentoxifylline was tapered completely over three months. This was followed by a flare of the condition. Pentoxifylline $400 \mathrm{mg}$ three times daily dose was reintroduced in addition to colchicine $0.5 \mathrm{mg}$ twice daily dose. Subsequently leg ulcers got resolved completely within three months. Pentoxifylline and colchicine were slowly tailed off over another three months and maintenance therapy of $400 \mathrm{mg}$ once daily and $0.5 \mathrm{mg}$ once daily respectively were continued for one year. As she did not experience any recurrent attack both drugs were omitted completely. She has been in complete remission for 13 months while off therapy (Figures $5,6,7 \& 8)$. This patient is still being followed up on a regular basis for possible relapses and treatment for her atrophic scars.

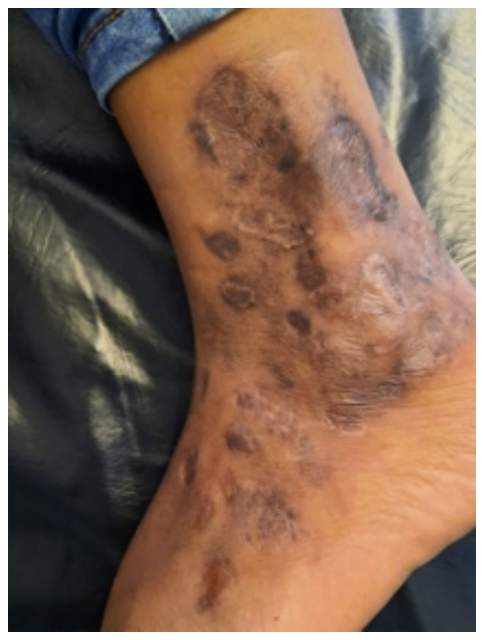

Figure 5: Healed lesions over lateral side of the ankle (left)

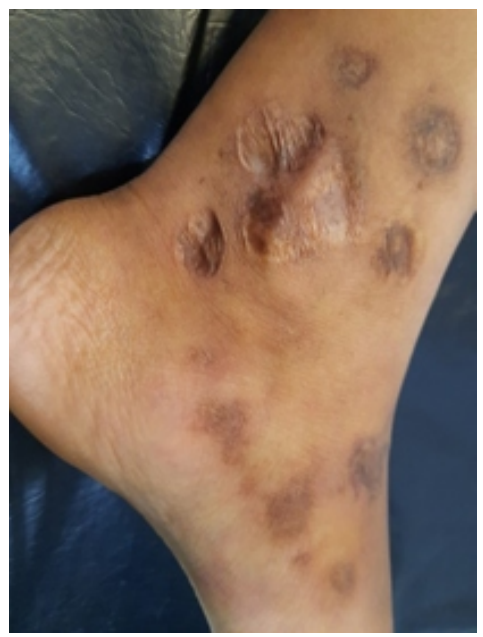

Figure 6: Healed lesions over medial side of the ankle (left)

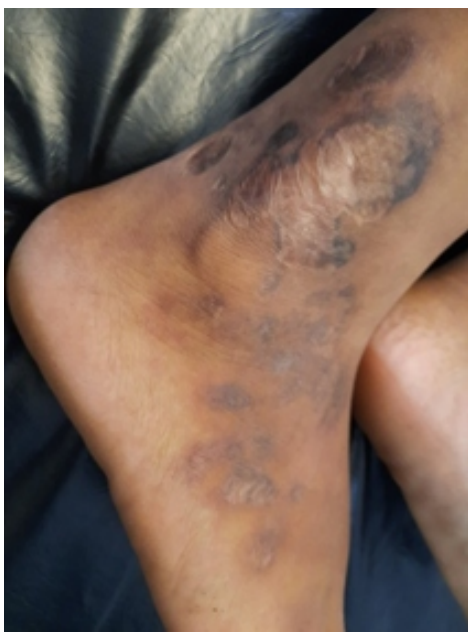

Figure 7: Healed lesions over lateral side of the ankle (right) 


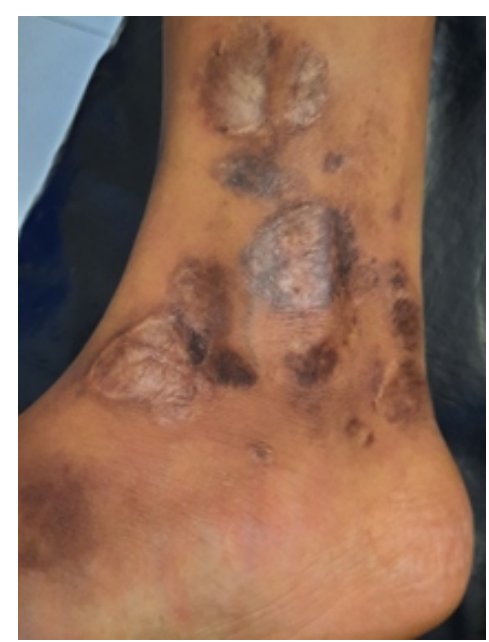

Figure 8: Healed lesions over the medial side of the ankle (right)

\section{Discussion}

$\mathrm{LV}$ is a rare dermatosis with an estimated incidence of 1 : 100,000 per year. Exact pathogenesis has not been clearly demonstrated yet. Most likely mechanism would be the formation of fibrin thrombi of the superficial dermal plexus. Hypercoagulability could be due to increased procoagulant activity, increased platelet activation, decreased fibrinolysis and decreased anticoagulant activity $(1,3)$. Differential diagnosis of LV includes other vascular diseases such as antiphospholipid syndrome and venous stasis systemic vasculitis, peripheral arterial disease, pyoderma gangrenosum. Therefore skin biopsy is mandatory (1). The characteristic histological findings in LV are mild perivascular lymphocytic infiltrates, extravasated red cells surrounding superficial dermal vessels with hyalinised walls and luminal fibrin deposition (2). Livedoid vasculopathy is seen in patients with and without identifiable coagulation abnormalities. Despite a thorough coagulation work up, even if any identifiable cause could not demonstrated, these patients must be followed up regularly in view of excluding antiphospholipid syndrome, cryoglobulinaemia or systemic lupus erythematosus. There are no standard therapeutic guidelines for the management of LV. Various treatment regimens have been designed to enhance the blood flow and avert the blood clotting, namely anti-platelets (e.g. pentoxifylline, which also alters red cell flexibility), anti-inflammatory agents (e.g. colchicine, dapsone and hydroxychloroquine), vasodilatory agents, anticoagulants (e.g. rivaroxaban), fibrinolytic therapies (danazol), immunosuppressants, miscellaneous (Intravenous immunoglobulin, hyperbaric oxygen) and supplements (folic acid, vitamin B6 and vitamin B12)(3). Recalcitrant LV often does not respond to aforementioned agents as a monotherapy. Thus, various combinations are used to achieve an early remission to prevent severe scaring and disfigurement. Persistent response to pentoxifylline and colchicine combination may indicate possible inflammation as an underlying mechanism of the ulcers.

\section{Conclusion}

This is a case of recalcitrant livedoid vasculopathy, which showed a sustained remission with pentoxifylline combined with colchicine. Further studies are required to recommend this combination as the first line therapy in livedoid vasculopathy to prevent severe scarring and disfigurement.

\section{References}

1. Warren WP. Cutaneous manifestations of Micro vascular Occlusion Syndromes. In: Bolognia J, Jorizzo J, Schaffer J (Eds). Dermatology. $3^{\text {rd }}$ edition. Amsterdam: Elsevier; 2012. p369-384.

2. Weedon D. Weedon's Skin Pathology. $3^{\text {rd }}$ edition. Edinburgh: Elsevier; 2010. p 788-790.

3. Vasudevan B, Neema S, Verma R. Livedoid vasculopathy: A review of pathogenesis and principles of management. Indian J Dermatol Venereol Leprol. 2016; 82: 478-88.

4. Evans JM, Jensen JD, Sami N. Successful treatment of livedoid vasculopathy with rivaroxaban: Case Report. JAAD. 2015; 1(6): 340-341.

5. Micieli R, Alavi A. Treatment for Livedoid Vasculopathy: A Systematic Review. JAMA Dermatol. 2018; 154(2): 193-202. 